\title{
Optimizing cancer survivorship in primary care: patient experiences from the Johns Hopkins Primary Care for Cancer Survivors clinic
}

\author{
Youngjee Choi ${ }^{1}$ (D) Elaina Parrillo ${ }^{2}$ Jennifer Wenzel ${ }^{2,3,4} \cdot$ Victoria F. Grabinski $^{3} \cdot$ Aamna Kabani $^{3} \cdot$ Kimberly S. Peairs $^{1,4}$
}

Received: 11 October 2021 / Accepted: 7 January 2022

(c) The Author(s), under exclusive licence to Springer Science+Business Media, LLC, part of Springer Nature 2022

\begin{abstract}
Purpose The optimal delivery of survivorship care, particularly within primary care, remains poorly understood. We established the Johns Hopkins Primary Care for Cancer Survivors (PCCS) clinic in 2015 to address care challenges unique to cancer survivors. To better understand the care from the PCCS clinic, we interviewed patients about their perception of care delivery, survivorship care, and care coordination.

Methods We conducted semi-structured interviews with adult survivors of any cancer type seen in the PCCS clinic. A priori and in vivo coding of verbatim transcripts was part of the thematic analysis.

Results Seventeen cancer survivors were interviewed (ages 37-78). Themes that emerged were (1) optimal care and (2) the PCCS experience. Subthemes respectively included the ideal role of the primary care provider (1), telehealth/ COVID-19 challenges and opportunities (1), patient-derived value from the PCCS clinic (2), and improving the PCCS model (2). Overall, PCCS patients expected and experienced high-quality, comprehensive primary care by providers with cancer survivorship expertise. Patients reported telehealth benefits and challenges for survivorship care during the COVID-19 pandemic.

Conclusions PCCS patients perceived receiving high-quality primary care and valued being seen in a primary care-based survivorship clinic. The PCCS clinic can serve as a model of primary care-based cancer survivorship.

Implications for Cancer Survivors Ideal primary care provider roles and care coordination are important factors for highquality survivorship care and can be provided by a specialized cancer survivorship clinic in primary care.
\end{abstract}

Keywords Cancer survivorship $\cdot$ Primary care $\cdot$ Care coordination

\section{Introduction}

In 2006, the Institute of Medicine (now National Academy of Medicine) highlighted the need for better coordinated care for cancer survivors in its report "From Cancer Care to

Youngjee Choi

ychoi47@jhmi.edu

1 Division of General Internal Medicine, Johns Hopkins University School of Medicine, Baltimore, MD, USA

2 Johns Hopkins University School of Nursing, Baltimore, MD, USA

3 Johns Hopkins University School of Medicine, Baltimore, MD, USA

4 Johns Hopkins Sidney Kimmel Comprehensive Cancer Center, Baltimore, MD, USA
Cancer Survivor: Lost in Transition" [1]. While a growing number of survivorship clinics have been designed to better address the needs of the over 16.9 million cancer survivors in the USA, the optimal delivery of survivorship care, particularly within primary care, remains poorly understood. Multiple challenges of providing survivorship care in the primary care context have been identified, including primary care providers (PCPs) being unclear about their role and responsibility in cancer-related care provision, a lack of designating survivorship as a distinct area of care to be addressed, and limitations in health system and information infrastructure for survivorship care [2, 3]. Evaluation of previously existing survivorship care models showed heterogeneity in models, with concerns around effective care coordination and adequate provider training [4]. This systematic review summarizing models of care for cancer survivors found limited evidence on how survivorship models of care have worked in real world settings [4]. We established the Johns Hopkins 
Primary Care for Cancer Survivors (PCCS) clinic in 2015 to address transition and care delivery challenges unique to cancer survivors [5]. Here we used a qualitative approach to explore perceptions of primary care, survivorship care, and care coordination in the PCCS clinic.

\section{Methods}

\section{Setting and participants}

The Johns Hopkins PCCS clinic has six general internists with expertise in cancer survivorship. Patients are seen at two general internal medicine practices, with one urban, hospital-based site and another suburban, ambulatory clinic. PCCS providers at both clinics additionally have non-cancer-related longitudinal clinics, thereby integrating PCCS patients into a general primary care clinic at both sites. While each PCCS provider has designated patient slots for new cancer survivor patients (1-4 per month depending on the provider), established cancer survivor patients are seen in any of the provider's clinic days along with general patients. We provided 447 visits across 184 PCCS patients in 2019, and 402 visits across 162 PCCS patients in 2020.

These clinics are also involved in primary care transformation initiatives to provide case management, behavioral health support, and pharmacy support to all patients. PCCS patients also have access to nursing and nutrition visits through our general medicine clinics.

Patients seen at the PCCS clinic are survivors of any cancer type and referred from our cancer center, other specialists, or are self-referred. They are seen either as (1) a consultation to address survivorship needs, with longitudinal primary care provided by a separate primary care provider (PCP) or (2) for establishment of primary care. Visits address any of the following topics: cancer surveillance, cancer screening, treatment-related effects, health promotion, comorbidity management, and palliative care as appropriate. Care coordination with the cancer center has been facilitated by access to oncology visit notes, which may provide information on upcoming or outstanding testing and unmet patient needs; coordination has also involved personal communication when needed between PCCS providers and cancer team members.

\section{Recruitment and data collection}

Participants were recruited from the first 230 patients seen in the Johns Hopkins PCCS clinic with input from PCCS clinicians regarding eligibility and health status. Eligibility criteria included speaking English, being able to independently answer questions, and speaking with enough clarity to be audio recorded and transcribed. We used purposive sampling to email patients approximately every other month in an iterative manner beginning April 2020 during the COVID-19 pandemic; recruitment of participants stopped when data saturation was confirmed. Interviews were conducted from May 2020 through February 2021.

\section{Analysis}

Interviews were conducted over the Zoom platform or by telephone (EP, VG, AK) following a semi-structured interview guide (Supplement). The interviews were audio-recorded and transcribed verbatim. To safeguard the anonymity and confidentiality of participants, names were removed from the transcripts. Thematic analysis of each transcript followed an approach described by Braun and Clarke and was informed by three coders (EP, VG, AK) [6]. The software package f4analyse 2.5.2 EDUCATION was used to assist in thematic analysis [7]. We used a consensus, or negotiated, coding approach to establish intercoder agreement. The three coders (EP, VG, and AK) coded and achieved consensus for the first three interview transcripts to establish a coding scheme. All subsequent transcripts were assigned to at least two coders, applying a similar consensus approach along with frequent meetings among the three coders to review and agree on codes $[8,9]$. Members of the team (EP, VG, AK, JW) met throughout the iterative coding process to review codes, the coding manual, and to discuss emerging patterns and themes. This process included repeated reading of the interview transcripts. The final synthesis and interpretation also included debriefing sessions to review representative quotes and themes and subthemes with additional team members (YC, $\mathrm{KP})$. This process elicited important feedback and facilitated discussions regarding analysis/interpretation, as well as finalized the themes and subthemes and data excerpts included.

\section{Results and discussion}

\section{Participant characteristics}

Of 78 cancer survivors emailed with an invitation, 17 agreed to participate and were interviewed. Interviewed survivors were mean 68 years in age (range 37-78), with median 2 years since diagnosis (IQR $1-4$ ). The most common cancer diagnoses were colorectal cancer and breast cancer (Table 1). Interviews were 20-90 min in duration. Interviews were shorter among participants who had only recently started to visit the PCCS clinic or who described relatively few oncology survivorship needs. All interviews were recorded and transcribed verbatim with the exception of one file where the recording data were lost and recreated from field notes and interviewer recall immediately post-interview. 
Table 1 Characteristics of interviewed PCCS patients

\begin{tabular}{|c|c|}
\hline Characteristic & $\begin{array}{l}\text { All patients } \\
n=17\end{array}$ \\
\hline Age - median (range) & $68(37-77)$ \\
\hline Female - no. $(\%)$ & $9(53)$ \\
\hline \multicolumn{2}{|l|}{ Race - no. $(\%)$} \\
\hline Caucasian & $16(94)$ \\
\hline African American & $1(6)$ \\
\hline \multicolumn{2}{|l|}{ Insurance - no. $(\%)$} \\
\hline Private & $11(65)$ \\
\hline Medicare & $6(35)$ \\
\hline \multicolumn{2}{|l|}{ Cancer type - no. (\%) } \\
\hline Colorectal & $6(35)$ \\
\hline Breast & $4(23)$ \\
\hline Prostate & $2(12)$ \\
\hline Gynecologic $^{\mathrm{a}}$ & $2(12)$ \\
\hline Other GI cancers ${ }^{\mathrm{b}}$ & $2(12)$ \\
\hline Leukemia & $1(6)$ \\
\hline \multicolumn{2}{|l|}{ Cancer stage at diagnosis ${ }^{\mathrm{c}}-$ no. $(\%)$} \\
\hline 1 & $4(25)$ \\
\hline 2 & $5(31)$ \\
\hline 3 & $5(31)$ \\
\hline 4 & $2(13)$ \\
\hline Cancer recurrence $^{\mathrm{d}}-$ no. $(\%)$ & $1(6)$ \\
\hline Local/regional & $1(6)$ \\
\hline Metastatic & $0(0)$ \\
\hline $\begin{array}{l}\text { Years between initial cancer diagnosis and initial } \\
\text { PCCS visit - median (IQR) }\end{array}$ & $2(1-4)$ \\
\hline \multicolumn{2}{|l|}{ Comorbidities - no. $(\%)$} \\
\hline Gastrointestinal disorder $^{\mathrm{e}}$ & $11(65)$ \\
\hline Psychiatric disease & $6(35)$ \\
\hline Hyperlipidemia & $6(35)$ \\
\hline Arthritis & $5(29)$ \\
\hline Hypertension & $5(29)$ \\
\hline Heart disease & $3(18)$ \\
\hline Lung disease & $1(6)$ \\
\hline Diabetes mellitus & $1(6)$ \\
\hline Thyroid disease & $1(6)$ \\
\hline $\begin{array}{l}\text { Body mass index at initial PCCS visit - median } \\
\text { (IQR) }\end{array}$ & $25.9(24.6-30.9)$ \\
\hline
\end{tabular}

${ }^{a}$ Endometrial and ovarian; ${ }^{b}$ pancreatic and gastroesophageal junction adenocarcinoma; ${ }^{c}$ the patient with leukemia was not included, as these staging groups do not apply; ${ }^{\mathrm{d}}$ cancers that were recurrent by the

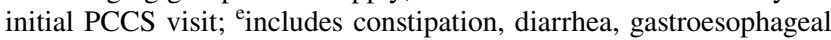
reflux disease, diverticular disease, delayed gastric emptying, and gastric arteriovenous malformation

\section{Interview themes}

In the 17 interviews with cancer survivors, we observed two overarching themes: optimal care and the PCCS experience. Themes, subthemes, and their descriptions can be found in Table 2 and are further discussed below. All references specific to physicians that could be potentially identifiable have been anonymized in brackets.

\section{Optimal care}

Cancer survivors discussed the optimal care that they expected and received as patients at the PCCS clinic. Optimal care included characteristics of the ideal role of the PCP and telehealth/COVID-19 challenges and opportunities. Patient expectations of the ideal role of the PCP included being cared for as a whole person, with physicians looking at overall health through a lens of cancer survivorship and coordinating care with the cancer team. Additionally, patients discussed their expectations and experiences of optimal care in the context of COVID-19 and receiving care through telehealth. Cancer survivors expected and experienced new challenges related to telehealth and COVID-19, such as fewer in-person clinic visits to optimize patient safety. However, telehealth also brought new opportunities, such as increased provider access through virtual visits.

\section{Ideal role of the PCP}

At the PCCS clinic, providers have the expertise to operate within both primary care and cancer survivorship care with the structure of a primary care team. Patients discussed that ideal PCPs were those that delivered comprehensive primary care, which for many patients included cancer survivorship care and care coordination.

Most patients expressed that their expectation was to receive high-quality primary care at the PCCS clinic:

"My primary interest was simply getting, you know, good internal medicine." - [P6, 68, female]

We observed that patients not only expected high-quality primary care, but also perceived receiving high-quality, comprehensive primary care from their providers:

"But [my PCCS physician] was very helpful and making sure that [they] can see the full picture of what all the different things that have happened to me and that are going on in my medical history, as opposed to like each of those different subspecialties that, that may or may not have the complete picture..." - [P2, 37, male].

"So I have one provider and what I like about [PCCS physician] is I can talk about everything. I usually go in there we go through each system" - [P7, 60, male].

Many patients also stated that their ideal PCPs, in delivering cancer survivorship care, would address cancerrelated issues and side effects from treatment, monitor for 
Table 2 Themes, subthemes, and participant quotes

\begin{tabular}{lll}
\hline Theme & Subtheme & Meaning of subthemes with participant quote examples \\
\hline Optimal care & Ideal role of the primary care provider & $\begin{array}{c}\text { Healthcare providers who delivered holistic primary care to patients. } \\
\text { For many patients, this included the ability to provide cancer } \\
\text { survivorship care and coordinate with the patient's other healthcare } \\
\text { providers } \\
\text { Example: "Well, I was primarily just looking for a primary care doc- } \\
\text { tor. And then learning more about how they, this particular practice } \\
\text { specializes in cancer or post cancer patients made it very attractive to } \\
\text { me and, so I think you know as far as my expectations go, I think you } \\
\text { know, I was very pleased to hear that and felt that there may be, you } \\
\text { know, a better understanding of my medical needs with a practice like } \\
\text { this."- [P15] }\end{array}$
\end{tabular}

Telehealth/COVID-19 challenges and opportunities How patients experienced COVID-19 and telehealth at the PCCS clinic in the context of cancer survivorship

Example: "My biggest, my biggest problem now, which I do consider a pretty big problem is, is the fear and the real end reality of contracting a communicable disease, which we don't have to mention the name of. And [my PCCS physician] has been, you know, been very helpful in that, you know, to have home healthcare instead of going in for blood work. I think was invaluable especially that was four weeks ago, four weeks ago is no time to be going into a medical facility. Not that today's that much better."- $[P 1]$

PCCS experience Meaning of the PCCS clinic for cancer survivors

The PCCS clinic helps patients shape their identity in terms of survivorship and serves as a home base for patients transitioning away from active cancer treatment in need of coordination. Establishing care at the PCCS clinic is particularly important for patients without an adequate primary care home

Example: "The team... that got me through the cancer, you know, gradually, you're being discharged... And I'm starting to get nervous because I've got all these things going on. And this person says they can't manage it and I can't, I don't know what's going on. I've never been through this before. And, you know, nobody else kind of knows. So it was kind of like 'Okay, well I gotta go somewhere where somebody can help me with all of these issues."'- [P7]

Improving the PCCS model of care

Patients sought more clarity about the role of the clinic, more capacity to accept new patients, and more involvement in cancer education and advocacy

Example: "The only concern that I sometimes have is when I have a small sort of thing that I used to take to my regular PCP, you know the upper respiratory issue or other you know issue, which are very real issues which do need treatment. I do feel a little bit inhibited by calling [my PCCS physician] because [my PCCS physician] has a lot of important obligations. I do understand I can call in and you know reach a nurse practitioner, but I think just determining where I go for minor things that may need care but don't rise to the level of [my PCCS physician's] degree of specialty is the one concern."- [P6]

recurrence, and use cancer as a context for providing care and recommendations:

"Well, my hope was that, that [my PCCS physician] indeed would kind of pay special attention to anything that could be related to symptoms related to my previous cancer diagnosis or if I have any complaints maybe particularly will be aware of that in terms of maybe recurrent cancer" - [P14, 55, female].

"So I reached out to [my PCCS physician's] practice as my as my treatment was winding down and started seeing [my PCCS physician] because I knew I was going to have some like, you know, side effects and after effects and I wanted to have a primary care physician who understood what my body had been through in treatment and would be able to kind of help me heal and, you know, move on from the disease" - [P11, 47, female].

Patients described their expectation that providers delivering cancer survivorship care had experience or specialization in cancer. Providers at the PCCS clinic met this 
expectation, which reassured patients about the care they received:

"Well, I was primarily just looking for a primary care doctor. And then learning more about how they, this particular practice specializes in cancer or post cancer patients made it very attractive to me and, so I think you know as far as my expectations go, I think you know, I was very pleased to hear that and felt that there may be, you know, a better understanding of my medical needs with a practice like this" - [P15, 68, female]. "That [PCCS physician] would have some experience, would have more experience than other internists, which is what I was going to end up with a primary care doctor with, with cancer survivors. Um, and I really didn't realize how much [my PCCS physician] does have which is very, made me very happy. It's been very reassuring" - [P1, 70, male].

In addition to the importance of receiving comprehensive primary care and cancer survivorship care, patients expressed the importance of their PCP coordinating care with their other healthcare providers and cancer team. Patients expected PCCS providers to coordinate and oversee their care:

"I guess when I saw [my PCCS physician] and heard [my PCCS physician], it kind of was bingo, in my mind, like I really need that primary care person again to kind of be the nucleus for all of my care, if that makes sense" - [P10, 59, female].

"What I was hoping, was that a certain amount of coordination of my care and a certain amount of insight into health conditions that I might have as a long term cancer survivor will be provided" - [P6, 68, female]. "...so it's going to be critical that this person can see everything and help me manage everything..." - [P7, 60, male].

One patient discussed their experience with care coordination, detailing that their PCCS provider was "in sync" with cancer team members and other healthcare specialists:

"But I mean, [healthcare specialist] referred to [PCCS physician]. [PCCS physician] referred to [healthcare specialist]. So I feel like that they are in sync. But I also feel like [my PCCS physician is] allowing the specialist to do his thing. And [PCCS physician] monitors what's going on" - [P10, 59, female].

Patients appreciated the ease of communication afforded by receiving care within a single medical system (e.g., between the Johns Hopkins Sidney Kimmel Cancer Center and the PCCS clinic). When talking about care coordination, many patients discussed that their PCCS provider was able to stay connected with the cancer team through the electronic medical record (EMR) Epic ${ }^{\mathrm{TM}}$ :

"I guess it's in Epic and whenever I go into [PCCS physician's] office [PCCS physician] is totally familiar with what the other doctors have, what's happened, because I see them actually more than I see [PCCS physician]. I see [PCCS physician] about every four months. I'd see them [the other doctors] every two months. So, and they're communicating in that way" - [P1, 70, male].

"I honestly I think they're doing that, you know, it's obvious to me because I've asked [PCCS physician] questions and [PCCS physician] sent me back messages, well I talked to your surgeon and talk with this person. I talked to that person. So, you know, I know that [there's] coordinating going on" - $[\mathrm{P} 4,72$, male].

When discussing their experience with what was going well regarding the coordination between the PCCS provider and cancer team, this patient described a sense of comfort knowing that they were a team:

"I think it's just a comfort knowing that that the team is there, you know, there's a great song out there called a "Safe Place to Land," I feel like the clinic and Hopkins is that my is my place. You know, so it's a comfort feeling" - [P13, 65, male].

PCCS physicians and their teams provided patients with the experience of optimal care. Patients expected and received comprehensive and high-quality primary care. For many patients, the primary care they received was in the context of being a cancer survivor. PCCS providers were specialized to deliver expert primary and cancer survivorship care to address the complex healthcare needs of cancer survivors. PCCS providers also stayed connected to other healthcare providers, including the patient's cancer team.

\section{Telehealth/COVID-19 challenges and opportunities}

Because all interviews were conducted in the midst of the COVID-19 pandemic, patients described telehealth/COVID19 challenges and opportunities within the context of the care they received as a cancer survivor. Telehealth was noted by some patients as an additional avenue to access care in ways that addressed distance barriers. One patient summarized the benefits of telehealth:

"The telehealth has actually been great. It would be great if telehealth could continue after this whole pandemic recedes, because for things like that where I would normally you know, have to go into the office and it just didn't make sense for such a minor thing to have to drive an hour, hour and a half, to go 
get seen. I would just go to an urgent care clinic" [P2, 37, male].

Patients also saw telehealth and home healthcare as a new opportunity for risk mitigation in the context of being a cancer survivor during COVID-19:

"[My PCCS physician] coordinates well with my other you know providers, like for example, I just had an appointment with [my PCCS physician] and you know [my PCCS physician] was easily able to see that I had been seen by another provider.... [PCCS physician] said no needs to come in, let's just do a telehealth visit. And so I really appreciated that especially given the pandemic" - [P8, 49, female].

Other patients were concerned about not being able to be seen by their clinic provider in-person more frequently. However, they balanced their concerns by considering their COVID-19 risk and safety rules created by the clinic to minimize risk for vulnerable patients:

"My biggest concern is that they are discouraging in-person visits right now, so I can't really go up there, but at the same time, it's probably for the best because like I was seen by my surgeon just last week, so it's not like I haven't had a physical exam in person" - [P11, 47, female].

In addition to experiences with telehealth, one patient described the anxiety cancer survivors experienced within the context of COVID-19 and how COVID-19 complicated an already challenging diagnosis and disease experience:

"I' $m$ in one of the most vulnerable groups. I consider myself in the triple whammy group....because I' $m$ a an old guy with cancer I think I need to go into health care facilities, a lot, which is another you know another health, health condition because I'm going to be exposed, I'd be exposed to other sick people. So I'm uh planning to do a lot of these [telehealth] visits" - [P1, 70, male].

In summary, the COVID-19 pandemic brought new challenges as some patients perceived optimal care as more frequent in-person visits. However, the pivot to telehealth allowed for access to care with a reduction in infection risk for the most vulnerable.

\section{PCCS experience}

The PCCS clinic experience had two sub-themes: patientderived value of the clinic and improvements to the PCCS model of care. Patients found the PCCS clinic meaningful in its ability to guide patients during their healthcare transition from active cancer treatment to cancer survivorship care. As a consequence, patients felt confident in their care and seemed to claim their identity as a cancer survivor. Additionally, patients receiving care at the PCCS clinic were able to offer suggestions for improvement based on their experiences at the clinic and needs as a cancer survivor.

\section{Patient-derived value of the clinic}

The value and importance defining the PCCS clinic for patients were having a healthcare practice that centralized patient care; addressed complex, primary care needs; shaped one's identity as a cancer survivor; and established confidence in care.

The PCCS clinic was particularly important as a "nucleus" for cancer survivors in times of transition. On being discharged from cancer treatment, some patients expressed a feeling of abandonment by their cancer team:

"Number one, when you are effectively discharged from something as intense as treatment for leukemia, it's almost a letdown. All of a sudden you're... something that has been a major factor in your life for months is suddenly not there, and it's almost as though you've been abandoned..." - [P3, 75, male].

Many survivors who used their oncologist for primary care needs during cancer treatment sought care at the PCCS clinic to fill this gap after treatment:

“...when I think of a primary care physician, I think of someone who really like I said, is holistic and knows your background, knows your history, knows what risk factors you have which you know what you need to be doing for prevention. And so I was kind of just relying on all of my specialists for that" - [P8, 49, female].

"The team... that got me through the cancer, you know, gradually, you're being discharged... And I'm starting to get nervous because I've got all these things going on. And this person says they can't manage it and I can't, I don't know what's going on. I've never been through this before. And, you know, nobody else kind of knows. So it was kind of like 'Okay, well I gotta go somewhere where somebody can help me with all of these issues"” - [P7, 60, male].

Prior to finding the PCCS clinic, some cancer survivors were unsure of where to find care in times of transition. For example, some felt guilt for using oncologists for primary care needs:

"I was okay with [my oncologist] being my primary care doctor but I realized for a lot of reasons, it wasn't 
practical. It wasn't fair for me to take her time from other patients" - [P1, 70, male].

Others could not find a primary care physician to coordinate complex cancer needs, or were disappointed when other physicians were not perceived to be sensitive to their cancer history:

“....after my cancer diagnosis, when I was trying to connect my oncologist at Hopkins up with my primary care doctor, my primary care doctor really didn't want to have anything to do with me because he told us that I was at a high risk category.... that other primary care physician wasn't interested in treating cancer survivors" - [P2, 37, male].

“... after going through cancer treatment, you're kind of fragile emotionally and physically, and the last thing I needed was a doctor who was going to be insensitive to the fact that I was really scared of any kind of recurrence, or I'm very aware of the fact that my risk for any subsequent cancer was much higher than a regular person" - [P11, 47, female].

Receiving care at the PCCS clinic helped some survivors shape their identity. For some, the clinic offered a survivorship community. For others, the clinic helped them establish normalcy after their cancer diagnosis:

"You realize going in there that most of the people around you have a common cause... Cancer patients, generally speaking, relate to each other... You know what people have been through so it's a good environment to walk into... you're not alone" - [P13, 65, male].

"I've gotten healthier and healed from the, you know, cancer treatment and everything... It's really starting to fade into my rear view" - [P11, 47, female].

Once established at the PCCS clinic, many cancer survivors expressed newfound confidence in their primary care and care coordination:

"I think what's changed is just peace of mind. I know someone's there. I can go look at my information I can ask questions and get answers .... that peace of mind just never existed in my life when it came to health care. So, so that's been great" - [P13, 65, male]. "I would say that I'm less anxious... I mean, I, I've always felt like I had a good support system, but now I feel like I have a really solid support system because I've got [PCCS physician]" - [P8, 49, female].

Although the majority of patients experienced newfound comfort from the clinic, some noted similarity to other primary care practices and felt responsibility for their own care coordination:
"Really my experience has been the same thing that you would expect from any other doctor visit" - [P6, 68 , female].

"I look at it more as a partnership... when I'm speaking with any physician or nurse practitioner, you know, I try to sort of weave everything together. Because I'm me and I know my body best, I feel like I can sort of summarize what's going on with me a little better than the doctor can who has goodness knows how many dozens, if not hundreds of patients" - [P12, 71, female].

Overall, the PCCS clinic was viewed as a support system by patients, influencing patient experiences on the journey of cancer survivorship and filling a gap in primary care needs after active treatment.

\section{Improving the PCCS model}

When asked about how the PCCS clinic could improve patient care, most cancer survivors commented on larger health system issues including difficulty scheduling through a centralized system or difficulty with their providers' ability to communicate across different EMRs. Clinic-specific suggestions for improvement included clarifying the role of the clinic for its patients, expanding access for new patients, and adopting a broader role in survivorship advocacy and education.

Some patients disclosed a hesitancy to contact their PCCS primary care physician with non-cancer-related primary care issues, which are well within the scope of the PCCS clinic. This highlights a need to better educate patients about the role of the clinic as a home for both cancer-related and general primary care needs:

"The only concern that I sometimes have is when I have a small sort of thing that I used to take to my regular PCP, you know the upper respiratory issue or other you know issue....I do feel a little bit inhibited by calling [my PCCS physician] because [my PCCS physician] has a lot of important obligations.... I think just determining where I go for minor things that may need care but don't rise to the level of [my PCCS physician's] degree of specialty is the one concern" - [P6, 68 , female].

In addition to clarifying the role of the PCCS clinic, several patients asked for more clarity in how to contact their clinic provider outside of a visit:

"I think that having something available either online or printed matter that would help people know when and where they might, you know, contact the clinic for routine things, things that may or may not be cancer 
treatment related would be helpful, almost like a calling triage card..." - [P6, 68, female].

Cancer survivors found the clinic in a variety of ways, often through their oncology team or a survivorship event. However, some patients were dissatisfied with the limited capacity of the PCCS clinic to accept new patients:

"Perhaps it would be helpful to have a list - I mean that they don't necessarily have to be part of the clinic, but doctors who maybe have more experience with oncology, or people who are cancer survivors. I literally don't think there's another doctor in that list that is accepting new patients, which is kind of distressing", - [P5, 68, female].

Additionally, a few patients wanted the PCCS clinic to expand its efforts in research and education and advocacy in the broader cancer community:

“And I don't know, I just think it would be really helpful to have more local support groups for cancer survivors... I had never met anybody my age who'd had this cancer so I had no idea, I was terrified when I was diagnosed..." - [P11, 47, female].

"It would be nice if the clinic maybe participated more in some of the things we're doing with the survivorship group..." - [P13, 65, male].

The PCCS clinic experience could be improved for future patients by clarifying the different cancer- and non-cancer-related care provided by PCCS providers, and by leading efforts in research and advocacy for cancer survivors. The majority of patients commented on an increased need for provider availability to accept new patients, demonstrating the value patients feel the clinic brings to their care as a cancer survivor.

\section{Discussion}

In interviewing 17 cancer survivors from the PCCS clinic, we found two main themes. The first was optimal care, with two important subthemes (1) the ideal role of the PCP and (2) telehealth in the context of the COVID-19 pandemic. The second main theme was the PCCS experience.

In considering the ideal role of the PCP, the American Academy of Family Physicians (AAFP) defines primary care as "...the provision of integrated, accessible health care services by physicians and their health care teams ... [to address] a large majority of personal health care needs" [10]. To achieve this, the PCP has traditionally held the role of "quarterback" for a patient's healthcare, managing and care coordinating for comorbidities that can involve multiple providers. While diabetes may come to mind for chronic conditions co-managed with subspecialists, cancer follows a similar pattern, where ongoing symptom and disease monitoring with other providers is essential. This concept of PCPs working in conjunction with other providers and being the "nucleus" that holds it all together was highlighted in our interviews with cancer survivors. We captured these important attributes described by patients as the ideal role of the PCP. While individual ideal physician behaviors such as "confident, empathetic, [and] humane" have been cited in the literature $[11,12]$, the ideal characteristics drawn from our interviews suggest more general qualities essential to the PCP role; they are in line with how the AAFP describes PCPs: "Primary care physicians advocate for the patient in coordinating the use of the entire health care system to benefit the patient" [10].

While most proposed models of cancer survivorship care are centered in oncology, including oncogeneralist models where PCPs with expertise in cancer work within the cancer center [12], a survivorship care model housed in primary care has inherent value. Not only is primary care used to coordinate care for multiple medical conditions, but having care centralized in primary care also allows for both cancer-related and all other (non-cancer-related) healthcare to be viewed in context of each other. The PCP can provide comprehensive care and see the patient as a whole individual, while accounting for the cancer history in evaluating symptoms and managing concerns. Having the infrastructure that a primary care team can provide further supports this holistic approach. This aspect was another ideal characteristic of the PCP described in interviews. While the interview themes highlighted the PCCS physicians' role, the interviews also reflected characteristics of the Patient Centered Medical Home (PCMH). The PCCS clinic was described as providing both comprehensive and coordinated care, as well as accessible services (through telehealth) _three defining aspects of the PCMH [13]. Patients also described being in partnership with PCCS physicians and the primary care team helping patients manage their own care, providing the "patient centered" aspect of the PCMH.

Our interviews were conducted during the COVID-19 pandemic, which highlighted both its challenges and opportunities to cancer survivors. While the literature has shown the risk to cancer patients from COVID-19 and delays in cancer-related care, the explosion of telehealth has been cited as one of the beneficial consequences of the pandemic [14, 15]. Similarly, our patients expressed their fears surrounding COVID-19 and the advantages of having telemedicine as an option during the pandemic. With state-mandated lockdowns beginning in March 2020, the PCCS general medicine clinic rapidly deployed telemedicine efforts, escalating from no telemedicine (0/316 total visits in the first week of March) to a peak of 273 weekly televisits (94\% of all visits) by May 2020 . The clinic offered in-person evaluations, but many patients preferred and continue to choose the video option when 
appropriate. Our PCCS physicians prioritized providing telehealth, recognizing its value to some of our most vulnerable patients, especially while care providers were still learning about COVID-19 transmission and susceptibility.

The main limitation of our study includes the small sample size. The small subset of PCCS patients who agreed to be interviewed may have been self-selecting, i.e., those who responded may have had the strongest feelings about the PCCS clinic and their care. However, we recruited participants until data saturation was confirmed, which suggests a consistency in the views and themes found in our analysis.

Patients overall expressed high satisfaction with the care received at the PCCS clinic, including through the pandemic. Given its success at our institution, we believe it could be a model of cancer survivorship in primary care for other programs [5]. While historically the literature has been sparse on how models of survivorship care work in real world settings [4], we believe the PCCS patient experiences outlined here and our prior description of the PCCS clinic can be a helpful roadmap [5]. To disseminate what we have learned during our first 6 years, we have been networking with other institutions interested in establishing parallel clinics. We also need to expand the PCP workforce that is knowledgeable and confident in providing survivorship care in order to meet growing demand. Increasing available educational resources for PCPs may be a first step to increase this capacity. We agree with patient observations: encouraging clinical research and physician advocacy in cancer survivorship will be important to demonstrate and promote the value of survivorship clinics and physician expertise in survivorship care. We hope that by taking these steps and incorporating patient recommendations as programs expand, models for cancer survivorship can be refined and expanded, and meet the needs of our growing population of cancer survivors.

\section{Conclusion}

The PCCS clinic is a unique model of cancer survivorship in primary care. Cancer survivors expected and experienced highquality, comprehensive primary care highlighted by expertise in cancer survivorship and care coordination with the cancer team. This level of care was delivered through the COVID19 pandemic, and patients reported the benefits of telehealth during this time. To increase access to this survivorship care delivery model within primary care, we believe similar clinics can be designed at other institutions, building on the strengths identified by patients while also addressing some of the current limitations; concurrently, efforts to increase the PCP workforce knowledgeable in survivorship care will be essential.

Supplementary Information The online version contains supplementary material available at https://doi.org/10.1007/s11764-022-01166-3.
Funding This work was supported by the Merck Foundation (grant number N022890).

Data availability The datasets generated during and/or analyzed during the current study are available from the corresponding author on reasonable request.

\section{Declarations}

Conflict of interest Drs. Youngjee Choi, Kimberly Peairs, and Jennifer Wenzel have received salary support from the Merck Foundation grant. The other authors declare they have no conflict of interest.

\section{References}

1. Hewitt M, Greenfield S, Stovall E. From cancer patient to cancer survivor: lost in translation. Washington, DC: National Academies Press.

2. Crabtree BF, Miller WL, Howard J, Rubinstein EB, Tsui J, Hudson SV, O'Malley D, Ferrante JM, Stange KC. Cancer survivorship care roles for primary care physicians. Ann Fam Med. 2020;18(3):202-9.

3. Rubinstein EB, Miller WL, Hudson SV, Howard J, O’Malley D, Tsui J, Lee HS, Bator A, Crabtree BF. Cancer survivorship care in advanced primary care practices: a qualitative study of challenges and opportunities. JAMA Intern Med. 2017;177(12):1726-32.

4. Halpern MT, Viswanathan M, Evans TS, Birken SA, Basch E, Mayer DK. Models of Cancer Survivorship care: overview and summary of current evidence. J Oncol Pract. 2015;11(1):e19-27.

5. Choi Y, Radhakrishnan A, Mahabare D, Patole S, Dy SM, Pollack CE, Berger ZD, Peairs KS. The Johns Hopkins Primary Care for Cancer Survivor clinic: lessons learned in our first 4 years. J Cancer Survivorship Res Pract. 2020;14(1):19-25.

6. Braun V, Clarke V. Using thematic analysis in psychology. Qual Res Psychol. 2006;3(2):77-101.

7. f4analyse 2.5.2 EDUCATION. https://www.audiotranskription. de/en/f4analyse/.

8. Campbell JL, Quincy C, Osserman J, Pedersen OK. Coding in-depth semistructured interviews. Sociol Methods Res. 2013;42(3):294-320.

9. Garrison D, Cleveland-Innes M, Koole M, Kappelman J. Revisiting methodological issues in transcript analysis: negotiated coding and reliability. Internet Higher Educ. 2006;9(1):1-8.

10. American Academy of Family Physicians. Primary care. https://www. aafp.org/about/policies/all/primary-care.html. Accessed 11 Aug 2021.

11. Bendapudi NM, Berry LL, Frey KA, Parish JT, Rayburn WL. Patients' perspectives on ideal physician behaviors. Mayo Clin Proc. 2006;81(3):338-44.

12. Nekhlyudov L. Integrating primary care in cancer survivorship programs: models of care for a growing patient population. Oncologist. 2014;19(6):579-82.

13. Agency for Healthcare Research and Quality. Defining the PCMH. https://pcmh.ahrq.gov/page/defining-pcmh. Accessed Jan 4, 2022.

14. Kutikov A, Weinberg DS, Edelman MJ, Horwitz EM, Uzzo RG, Fisher RI. A war on two fronts: cancer care in the time of COVID19. Ann Intern Med. 2020;172(11):756-8.

15. Mayor S. COVID-19: impact on cancer workforce and delivery of care. Lancet Oncol. 2020;21(5):633.

Publisher's note Springer Nature remains neutral with regard to jurisdictional claims in published maps and institutional affiliations. 